www.jmscr.igmpublication.org

Impact Factor 5.84

Index Copernicus Value: 71.58

ISSN (e)-2347-176x ISSN (p) 2455-0450

crossref DOI:_https://dx.doi.org/10.18535/jmscr/v5i9.01

\title{
Topical Corticosteroids - A Great Challenge to Avoid Misuse on Face
}

\author{
Authors \\ Dr Sandhya Chauhan', Dr Ashok Garg', Dr Prem Lal ${ }^{3}$, Dr Ajay Ahluwalia ${ }^{4}$ \\ ${ }^{1}$ Dermatologist, ${ }^{2}$ Pediatrician, ${ }^{3} \mathrm{MD}$ Community Medicine, ${ }^{4}$ Radiologist \\ Mahatma Gandhi Medical Service Complex, Khaneri, Rampur-172001, Shimla (HP) \\ Corresponding Author \\ Dr Ashok Garg \\ Email: ashokgarg44@gmail.com Contact: 9459373371, 9817059771 \\ Address: House no. 35, Ward no. 2, Village Anukalan, PO/Teh/ Distt. Hamirpur (HP)
}

\section{Abstract}

Introduction: Topical corticosteroids (TCS) are the most commonly prescribed topical drugs not only by dermatologists but also by non-dermatologists. Over the counter availability, irrational prescription and prolonged use by patients has resulted in their misuse. Steroid rosacea, hypertrichosis, steroid dependent face $(S D F)$, atrophy and acneform eruptions are some of facial side effects of these drugs.

Objective: To know clinico-epidemiological profile of patients misusing the TCS over face. To determine adverse effects, source of prescriptions, commonly misused preparations of TCS.

Methods: This is an observational study conducted over a period of six months from January to June 2016. Patients misusing TCS for more than one month and who presented with at least one side effect over the face were enrolled for this study. A detailed history regarding clinico-epidemiological parameters, duration of misuse, source, preparation and reason for use of TCS was taken. Adverse side effects were noted down and clinical photographs of patient were also taken after their consent.

Results: A total of 412 patients including 315 females and 97 males were studied. Majority of patients were in the age group of 11-40 years. Duration of application of TCS was <6 months in majority, longest being 5 years. Nearly $73.3 \%$ of the patients were using it on a daily/intermittent basis. Most commonly misused TCS was betamethasone in 37.9\% followed by clobetasole in 32.3\%. Indications for using TCS in descending order were; acne (31.5\%), fairness cream (23.8\%), pigmentation (19.9\%), tinea faci (10.4\%), facial dermatitis $(5.6 \%)$ and various other dermatoses of face (8.8\%). TCS were taken after the advice of chemists/pharmacists in $38.8 \%$, friends and relatives $24.8 \%$, paramedical persons $20.34 \%$, nondermatologists $12.9 \%$ and dermatologists 3.1\%. Commonest side effect was acneform eruptions and other side effects were SDF, rosacea and rosacea diathesis, hypertrichosis, dyspigmentation, infections and perioral dermatitis.

Conclusion: From our study it was concluded that misuse of TCS is very prevalent among young females in our setup. Immediate relief of signs and symptoms and unawareness about their side effects are the main reasons for their misuse. Further, over the counter availability, irrational prescriptions and poor access of patients to dermatologists worsen the abuse. Although it's a great challenge to overcome this problem but general public awareness, patient education and medical/paramedical personnel sensitization can help to some extent.

Key Words: Steroids, Facial abuse, Rosacea, Hypertrichosis, Acne. 


\section{INTRODUCTION}

Topical corticosteroids (TCS) became the main therapeutic tool for dermatologists with the introduction of first steroid 'hydrocortisone' in 1951 and super potent steroid in 1974. ${ }^{[1]}$ Since then, number of unwanted dermatosis emerged with the introduction of more number of higher potency TCS. The clinical effects as well as some side effects of steroids are due to anti inflammatory, anti proliferative and immunosuppressive effects. ${ }^{[2]}$ Topical steroids are misused both by prescribing personnel and patient themselves, as it gives instant relief to signs and symptoms. ${ }^{[3]}$ Some individuals use TCS without prescription as fairness, anti-acne and as general purpose cream. Steroids acne, rosacea, hypertrichosis and topical steroid dependent face (TSDF) are some facial side effects encountered by dermatologists in their routine practice. ${ }^{[3]}$

In India, the annual sales figure of TCS was 14 billion rupees in 2013, which accounts for almost $82 \%$ of total dermatological product sale in the country. ${ }^{[4]}$

As per the information available on the Central Drugs Standard Control Organization (CDSCO), TCS are widely practiced in India without any specific indication. ${ }^{[5]}$. Not only developing countries like India and Africa but even developed countries like United States of America is also facing the problem of TCS abuse. ${ }^{[2]}$

\section{MATERIAL AND METHODS}

This observational was study conducted among outdoor patients at MGMSC Khaneri, Rampur: a peripheral health institute of Himachal Pradesh over a period of six months (January to June 2016). A total of 412 patients irrespective of age/sex; who had history of TCS misuse over face for more than one month and who had developed at least one side effect of TCS misuse over the face were enrolled for this study. A detailed history regarding clinico-epidemiological parameters, duration of abuse, prescribing personnel, preparation of TCS and indication for use of TCS was taken. All adverse side effects were noted down and clinical photographs of the patient were also taken after their consent. Patients having co-morbidities that could cause similar changes to TCS side effects like cushing's syndrome, thyroid disorders, polycystic ovaries were excluded. Finally data was compiled and evaluated as shown in results.

\section{RESULTS}

Out of total 412 patients, 97 were males and 315 were females with male to female ratio 0.37. Age of the patients varied from 5 to 54 years; most of the patients were between the age group of 11-40 years. Further, $39.3 \%$ patients were from 11-20 year age group, $37.7 \%$ from 21-30 years and $12.13 \%$ patients from 31-40 year age group (Figure1). Majority (75\%) of the patients were not only well educated but also belonged to middle to upper class families. As per qualification status, $48.8 \%$ patients were educated upto secondary level, $30.6 \%$ upto tertiary level and while $20.6 \%$ patients were illiterate or had primary level education. Reasons for use of TCS in descending order were; acne (31.5\%), fairness cream (23.8\%), pigmentation $(19.9 \%)$, tinea faci $(10.4 \%)$, facial dermatitis $(5.6 \%)$ and various other dermatoses of face $(8.8 \%)$ (Figure2).

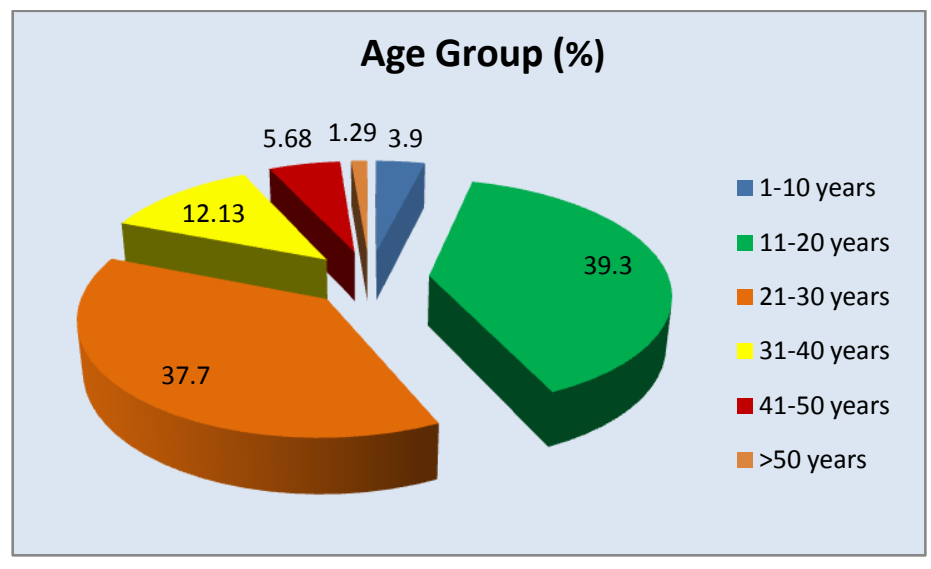

Figure 1: Patient distribution in different age groups 


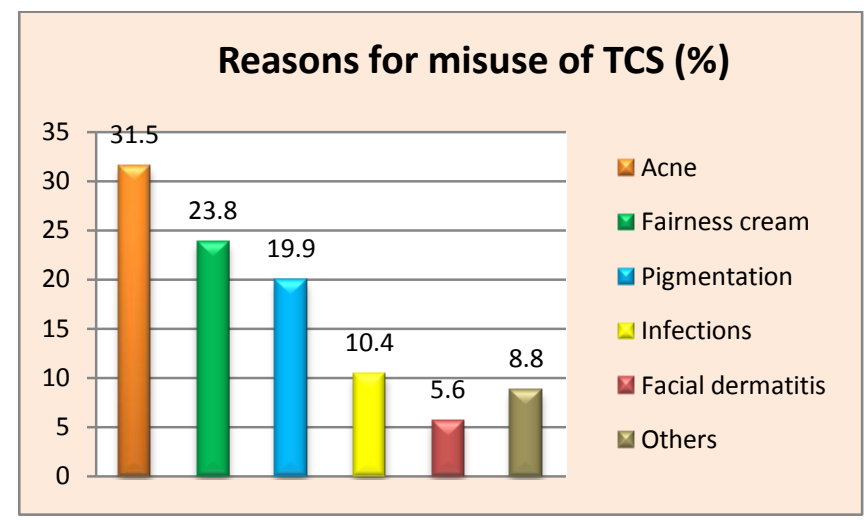

Figure 2: Reasons for misuse of TCS over face

Duration of application of TCS was $<6$ months in majority, longest being 5 years. Nearly $73.3 \%$ of the patients were using it on a daily/intermittent basis while the remaining $26.7 \%$ patients were using TCS in a frequency of two or more than two times a day. Sources of steroid prescription were chemists/pharmacist (38.8\%), self medication as advised by friends/relatives in $24.7 \%$, paramedical staff $20.34 \%$, non-dermatologists $12.9 \%$ and dermatologists in $3.15 \%$ (Figure 3). All patients were unaware about the side effects of TCS. Commonest observed side effect was acneform eruption (Figure 4) followed by steroid dependent face (SDF). Other side effects were steroid rosacea, hirsutism, (Figure 6, 4) dyspigmentation, perioral dermatitis and various infections (warts, herpes simplex, tinea incognito and pyoderma) (Figure 5). Combination formulations (i.e. steroid with antibacterial and antifungal agents and Kligman formula) were used by the majority (58\%) of patients. Most common TCS used by our patients was betamethasone $(37.9 \%)$ followed by clobetasole $(32.3 \%)$. Other misused TCSs were mometasone, flucinolone, fluticasone and desonide. PANDERM, BETNOVATE, CLOBENG, MELACARE and MELALITE were main brands misused by the most of the patients.

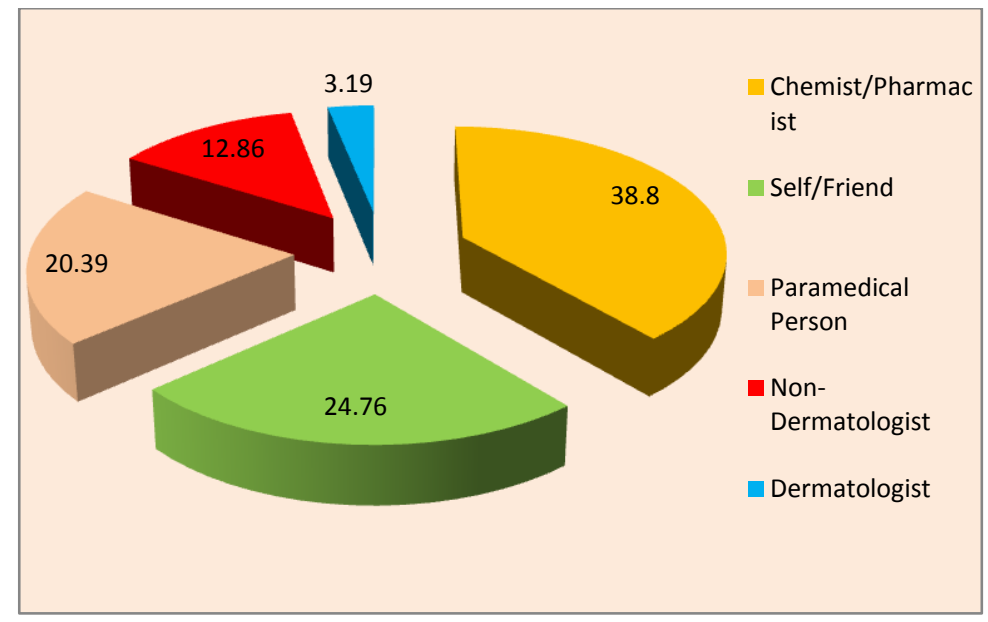

Figure 3: Person prescribing the TCS

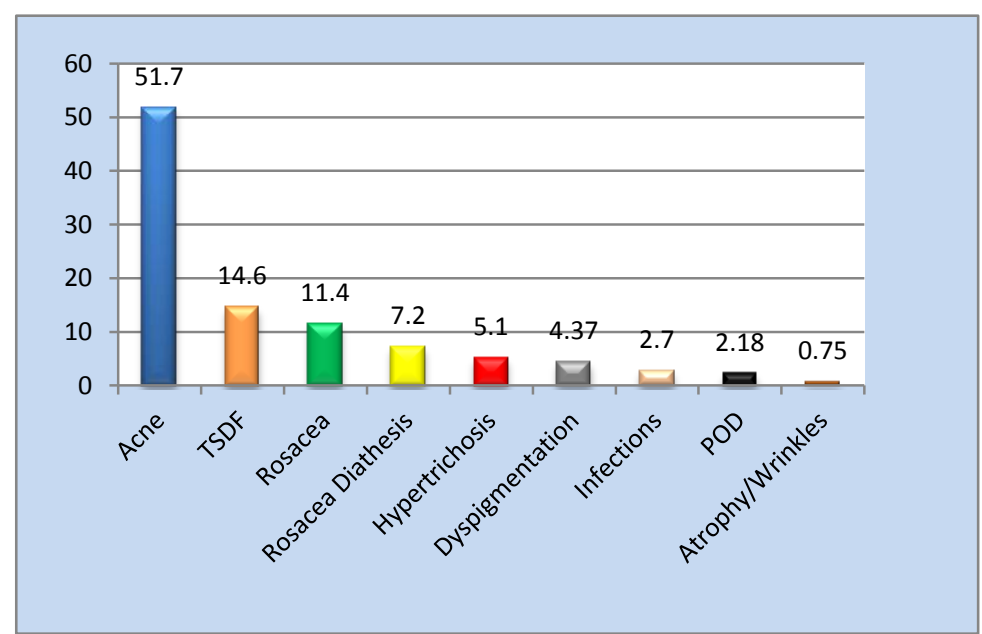

Figure 5: Observed side effects of TCS over face.

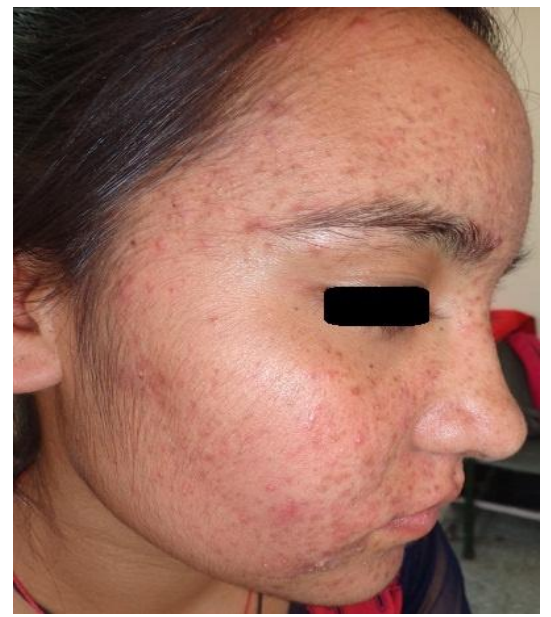

Figure 4: Steroid acne and hypertrichosis

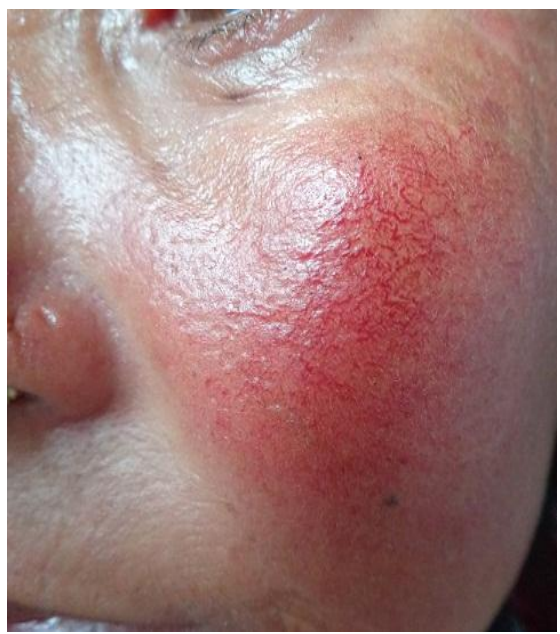

Figure 6: Steroid induced rosacea 


\section{DISCUSSION}

TCS abuse has become a common problem faced by dermatologist in different parts of the world. ${ }^{[6,7,8]}$ In 2006, Nnoruka et al reported that TCS are misused as depigmenting agents over the face in dark-skinned individuals. ${ }^{[8]}$ In India, the first case series on TCS abuse was published in 2006 and since then, numerous case studies have described TCS abuse over face. ${ }^{[9]}$ In our study females' outnumbered males, this is in accordance with other studies. ${ }^{[10,11]}$ In our study, maximum number of patients belonged to the age group of 11-20 years, while some studies shows 20-30 year age group more prone for TCS abuse. Acne was the most common indication of steroid application; hence, the predominance of younger age group in our study is easily explained and this is also reported in a study by Manchanda et al. ${ }^{[12]}$ Most of the subjects were using potent TCs in our study, which is in concordance with prior studies from other countries. ${ }^{[13]}$ Betamethasone alone or in combination was by far the most common corticosteroid used by our patients and same has been reported by Sharma et al. ${ }^{[4]}$ BETNOVATE, PANDERM and CLOBEN-G were the commonly misused brand names. Mometasone, hydroquinone and tretinoin containing skin-lightening formulas are very popular and very easily available in the market. This product with variable brand names (melacare, melanorm and melalite) was commonly abused in our study in agreement with study by Saraswat, et al. ${ }^{[7]}$

Chronic use of TCS often produces numerous side effects, which are usually seen after 6 months or more of continuous use of steroids but duration can vary with potency. ${ }^{[9]}$ Initial phase of TCS abuse may demonstrate erythematous papules to papulopustular eruptions. This is followed by inflammed, edematous lesions with papules, pustules, and nodules. ${ }^{[9]}$ Discontinuation of TCS after prolonged use results in severe rebound erythema, burning sensation and scaling on the face. ${ }^{[14]}$ TCS induced rosacea like dermatitis (TCIRD) is defined as a condition arising from prolonged use and the rebound phenomenon, which appears after discontinuation of TCS on the face. ${ }^{[15]}$ Various other terms have been used by authors describing this entity such as topical steroid dependent face (TSDF), red face syndrome, steroid dermatitis resembling rosacea (SDRR) and steroid rosacea. ${ }^{[14]}$ TSDF is a new entity characterized by erythema or burning sensation or pruritus on attempted cessation of TCS application. ${ }^{[15]}$ Persistent use of TCS can also lead to complications such as epidermal atrophy, degeneration of dermal structure with decrease collagen synthesis, rosacea, and susceptibility to superficial bacterial, viral and fungal infections. ${ }^{[14]}$ Almost all these adverse effects were observed in our patients.

To overcome the side effects of TCS, slow tapering by decreasing frequency or switching to lower potent steroid has to be done. Other effective drugs like low dose doxycycline, tetracycline and azithromycin can be prescribed. ${ }^{[16]}$ Recently topical tacrolimus and pimecrolimus reported to be effective drugs. ${ }^{[17,18]}$ Ideally no steroid is safe for face but where necessary, a less potent TCS should be prescribed for shorter duration only if specifically indicated. And it is very essential to educate patient about side effects and dependency in order to prevent the consequences of misuse. This kind of awareness among patients, non-dermatologists, chemists/pharmacists and paramedical staff and patient is highly essential to overcome the problem of TCS abuse.

\section{CONCLUSION}

From our study it was concluded that misuse of TCS is very prevalent among young females in our setup. Immediate relief of signs and symptoms and unawareness about their side effects are the main reasons for their misuse. Further, over the counter availability, irrational prescriptions and poor access of patients to dermatologists worsen the abuse. Although it's a great challenge to overcome this problem but general public awareness, patient education and medical/ 
paramedical personnel sensitization some recommendations to deal with this problem.

\section{REFERENCES}

1. Dey VK. Misuse of topical corticosteroids: A clinical study of adverse effects. Indian Dermatology Online Journal 2014;5:4

2. Mishra AK, Saraswat D. Topical Corticosteroid Abuse in Dermatology. IOSR Journal of Dental and Medical Sciences 2016:15(7);110-114.

3. Sharma R, Abrol S, Wani M. Misuse of topical corticosteroids on facial skin: A study of 200 patients. Dermatol Case Rep.2017;11(1):5-8.

4. Verma SB. Sales, status, prescriptions and regulatory problems with topical steroids in India. Indian J Dermatol Venereol Leprol 2014;80:201-03.

5. Fixed Dose Combinations approved By DCG (I) since 1961 till July, 2014. Central Drugs Standard Control Organization, India.

6. Al Dhalimi MA, Aljawahiry N. Misuse of topical corticosteroids: A clinical study in an Iraqi hospital. East Mediterr Health J2006;12:847-51.

7. Saraswat A, Lahiri K, Chatterjee M, Barua S, Coondoo A, Mittal A, et al. Topical corticosteroid abuse on the face: A prospective, multicentric study of dermatology outpatients. Indian J Dermatol Venereol Leprol 2011;77:160-6.

8. Nnoruka E, Okoye O. topical steroid abuse: Its use as a depigementing agent. J Natl Med Assoc 2006;98:934-9.

9. Rathi S. Abuse of topical steroid as cosmetic cream: A social background of steroid dermatitis. Indian $\mathrm{J}$ Dermatol 2006;51:154-5.

10. Ambika H, Vinod CS, Yadalla H, Nithya $\mathrm{R}$, Babu AR. Topical corticosteroid abuse on the face: A prospective study on outpatients of dermatology. Our Dermatol Online 2014;5:5-8.

11. Hameed AF. Steroid Dermatitis Resembling Rosacea: A Clinical Evaluation of 75 Patients. ISRN Dermatol 2013;49:1376.

12. Manchanda K, Mohanty S, Rohatgi PC. Misuse of topical corticosteroids over face: A clinical study. Indian Dermatol Online J 2017;8:186-91.

13. Keane FM, Munn SE, Taylor NF, du Vivier AW. Unregulated use of clobetasol propionate. Br J Dermatol 2001;144:109596.

14. Bhat YJ, Manzoor S, Qayoom S. Steroid induced rosacea. A clinical study of 200 patients. Indian J Dermatol 2011;56:30-2.

15. Lahiri K, Coondoo A. Topical Steroid Damaged/Dependent Face (TSDF): An Entity of Cutaneous Pharmacodependence. Indian J Dermatol.2016;61:265-272.

16. Rosso JQ. Management of Papulopustular Rosacea and Perioral Dermatitis with Emphasis on Iatrogenic Causation or Exacerbation of Inflammatory Facial Dermatoses: Use of Doxycycline-modified Release 40mg Capsule Once Daily in Combination with Properly Selected Skin Care as an Effective Therapeutic Approach. J Clin Aesthet Dermatol. 2011;4:20-30.

17. Lee DH, Li K, Suh DH. Pimecrolimus $1 \%$ cream for the treatment of steroid-induced rosacea: an 8-week split-face clinical trial. Br J Dermatol. 2008;158:1069-76.

18. Chu CY. An open-label pilot study to evaluate the safety and efficacy of topically applied pimecrolimus cream for the treatment of steroid-induced rosacealike eruption. J Eur Acad Dermatol Venereol. 2007;21:484-90. 